\title{
On stochastic recursive equations and infinite server queues
}

\author{
Eitan Altman \\ INRIA, BP93, 06902 Sophia Antipolis, France
}

\begin{abstract}
The purpose of this paper is to investigate some performance measures of the discrete time $\mathrm{G} / \mathrm{G} / \infty$ queue under a general arrival process. We assume more precisely that at each time unit a batch with a random size may arrive, where the sequence of batch sizes need not be i.i.d. All we request is that it would be stationary ergodic and that the service duration has a phase type distribution. Our goal is to obtain explicit expressions for the first two moments of number of customers in steady state. We obtain this by computing the first two moments of some generic stochastic recursive equations that our system satisfies. We then show that these class of recursive equations allow to solve not only the $G / P H / \infty$ queue but also a network of such queues. We finally investigate the process of residual activity time in a $G / G / \infty$ queue under general stationary ergodic assumptions, obtain the unique stationary solution and establish coupling convergence to it from any initial state.
\end{abstract}

Keywords: Stochastic processes/Queueing theory.

\section{INTRODUCTION}

Most explicit expressions for performance measures in queueing networks are known under independence assumptions on the driving processes (service and interarrival times). An interesting challenge is to obtain explicit expressions for the case in which the independence is relaxed and only stationarity and ergodicity of some components of the driving sequences are assumed. One line of research that allows to handle stationary ergodic sequences is based on identifying measures that are insensitive to correlations. For example, the probability of finding a $\mathrm{G} / \mathrm{G} / 1$ queue nonempty is just the ratio between the expected service time and the expected interarrival time (which follows directly from Little's Law). The expected cycle duration in a polling system (under fairly general condition) too, depends on the interarrival, service and vacation times only through their expectations under general stationary ergodic assumptions (see e.g. [5]). An example of performance measures that depend on the whole distribution of service times but is insensitive to correlations is the growth rate of number of customers or of sojourn time in a (discriminatory) processor sharing queue in overload [4], [13].

In this paper we study a queueing problem under a stationary ergodic arrival process, in which the correlations indeed influence the performance but in which despite the dependence between arrival times, explicit expressions are obtained for the two first moments of the stationary number of customers. More precisely, we study the discrete time G/G/ $\infty$ queue in which at each time unit a random a batch with a random size may arrive, where the sequence of batch sizes is stationary ergodic and service durations have a phase type distribution.

We first compute the two moments of some generic stochastic recursive equations that our system satisfies. These are simplified versions of stochastic recursions introduced in [2] which already enabled us to study polling systems [2], [12] and queues with vacations [2] in which vacation times are correlated, and are related to branching process with migration [1]. Yet this is the first time that generic explicit expressions are derived for the first two moments of such equations.

We then show that these class of recursive equations allow to solve not only the $G / P H / \infty$ queue but also a network of such queues.

We finally investigate the process of residual activity time in a $G / G / \infty$ queue under general stationary ergodic assumptions, obtain the unique stationary solution and establish coupling convergence to it from any initial state.

The infinite server queue which is the topic of our paper has had various applications in teletraffic and in networking modeling. The output process of an $\mathrm{M} / \mathrm{GI} / \infty$ queue has been used to model long range dependent traffic, c.f. in video applicationskruntz. In [17] the connectivity of ad-hoc networks on a line has been considered. The distribution of distance covered by a connected set of mobiles has been shown to correspond to a busy period in the $G I / G I / \infty$ queue and its distribution was computed for various channel conditions. Furthermore the distribution of the number of connected mobiles has been computed using its correspondence to the number of customers served in a busy period of a $G I / G I / \infty$ queue. 
Finally, the infinite server queue has also been used in the context of communication networks and distributed computer systems, see e.g. [14].

The structure of the paper is as follows. We introduce in Section II generic stochastic recursive equation corresponding to a branching type process in non Markov random environment with migration. The first and second moments of the corresponding state variables are introduced in Section III. The expressions obtained are shown to further simplify for specific Markovian dynamics that creates the correlation. This allows us to derive in Section IV explicit performance measures for the $G / P H / \infty$ discrete time queue and to provide a numerical example that illustrates the role of correlation. An extension to a whole network of infinite server queues ends this section. Further stability results for the $\mathrm{G} / \mathrm{G} / \infty$ queue are presented in Section V followed by a concluding section.

\section{THE MODEL}

Consider a column vector $Y_{n}$ whose entries are $Y_{n}^{i}, i=$ $1, \ldots, N$ where $Y_{n}^{i}$ take values on the nonnegative integers $\mathrm{Z}^{+}$. Consider the following stochastic recursive equation:

$$
Y_{n+1}=A_{n}\left(Y_{n}\right)+B_{n}
$$

where the $i$ the element of the column vector $A_{n}\left(Y_{n}\right)$ is given by

$$
\left[A_{n}\left(Y_{n}\right)\right]_{i}=\sum_{j=1}^{N} \sum_{k=1}^{Y_{n}^{j}} \xi_{j i}^{(k)}(n)
$$

where $\xi^{(k)}(n), k=1,2,3, \ldots, n=1,2,3, \ldots$ are i.i.d. random matrices of size $N \times N$. Each of its element is a nonnegative integer. Denote $E\left[\xi_{i j}^{(k)}(n)\right]=\mathrm{A}_{j i}$. The $N$ dimensional vector $B_{n}$ is a stationary ergodic stochastic whose entries $B_{n}^{i}, i=1, \ldots, N$ are nonnegative integers.

$A_{n}(y)$ has a divisibility property: if for some $k, y=$ $y^{0}+y^{1}+\ldots y^{k}$ where $y^{m}$ are integers, then $A_{n}(y)$ can be represented as

$$
A_{n}(y)=\sum_{i=0}^{k} A_{n}^{(i)}\left(y^{i}\right)
$$

where $\left\{A_{n}^{(i)}\right\}_{i=0,1,2, \ldots, k}$ are i.i.d. with the same distribution as $A_{n}(\cdot)$. Note also that $A_{n}(0)=0$. The divisibility property allows us to use the framework of [2] to characterize the distribution of $Y_{n}$ and its limiting behavior.

We shall understand below $\prod_{i=n}^{k} A_{i}(x)=x$ whenever $k<n$, and $\prod_{i=n}^{k} A_{i}(x)=A_{k} A_{k-1} \ldots A_{n}$ whenever $k>$ $n$.

We note that although (1) is not linear in $Y_{n}$, it is linear in expectation; if we let $y$ be a column vector then

$$
E\left[A_{n}(y)\right]=\mathrm{A} y .
$$

Moreover, we have for $j>1$ by Wald's equation

$$
E\left[\left(\prod_{i=1}^{j} A_{j}\right)(y)\right]=\mathrm{A}^{j} y
$$

We make the following assumptions throughout the paper:

A1: $\|\mathrm{A}\|<1$ and $E\left[\log \left\|B_{0}\right\|\right]<\infty$ where $\|\mathrm{A}\|$ stands for the largest absolute value of the eigenvalues of $A$ and where $\left\|B_{0}\right\|$ stands for the maximum absolute value of the elements of $B_{0}$.

Note that by Jensen's inequality, a sufficient for $E\left[\log \left\|B_{0}\right\|\right]<\infty$ is that $E\left[\left\|B_{0}\right\|\right]<\infty$.

We recall the following property of our system:

Theorem 1: (i) For $n>0, Y_{n}$ can be written in the form $Y_{n}=$

$$
\sum_{j=0}^{n-1}\left(\prod_{i=n-j}^{n-1} A_{i}^{(n-j)}\right)\left(B_{n-j-1}\right)+\left(\prod_{i=0}^{n-1} A_{i}^{(0)}\right)\left(Y_{0}\right)
$$

(ii) there is a unique stationary solution $Y_{n}^{*}$ of (1), distributed like

$$
Y_{n}^{*}={ }_{d} \sum_{j=0}^{\infty}\left(\prod_{i=n-j}^{n-1} A_{i}^{(n-j)}\right)\left(B_{n-j-1}\right), \quad n \in Z,
$$

The sum on the right side of (6) converges absolutely $P$ almost surely. Furthermore, for all initial conditions $Y_{0}$, $\left\|Y_{n}-Y_{n}^{*}\right\| \rightarrow 0, P$-almost surely on the same probability space. In particular, the distribution of $Y_{n}$ converges to that of $Y_{0}^{*}$ as $n \rightarrow \infty$.

Proof. (5) is obtained by iterating (1). Theorem 2 and Lemma 1 in [2] imply (ii).

\section{FIRST AND SECOND MOMENTS}

Denote by $y_{i}$ and $y_{i}^{(2)}$ the first and second moment of the $i$ th element of $Y_{n}^{*}$. Denote $\operatorname{cov}(Y)_{i j}=$ $E\left[\left(Y_{0}^{*}\right)_{i}\left(Y_{0}^{*}\right)_{j}\right]-y_{i} y_{j}$. Let $b_{i}$ and $b_{i}^{(2)}$ denote the two first moments of $B_{n}^{i}$. Denote $\operatorname{cov}(\xi)_{j k}^{i}=E\left(\xi_{i j}^{(0)} \xi_{i k}^{(0)}\right)-\mathrm{A}_{j i} \mathrm{~A}_{k i}$ and define the following $N \times N$ matrices:

$\mathcal{B}(k)$ is the matrix whose $i j$ th entry equals $E\left[B_{0}^{i} B_{k}^{j}\right]$, where $k$ is an integer.

$\hat{B}$ is the matrix whose $i j$ th entry equals $b_{i} b_{j}$, $\operatorname{cov}(B)$ is the matrix whose $i j$ th entry equals $E\left[B_{0}^{i} B_{0}^{j}\right]-$ $b_{i} b_{j}$.

Define $\hat{\mathcal{B}}(k):=\mathcal{B}(k)-\hat{B}$. 


\section{A. General results}

Theorem 2: (i) The first moment of $Y_{n}^{*}$ is given by

$$
E\left[Y_{0}^{*}\right]=(I-\mathrm{A})^{-1} b,
$$

(ii) Assume that the first and second moments $b_{i}$ and $b_{i}^{(2)}$, s are finite. Define $Q$ to be the matrix whose (ij)th entry is

$$
Q_{i j}=\sum_{k=1}^{N} y_{k}\left(\operatorname{cov}(\xi)_{i j}^{k}\right) .
$$

Then the matrix $\operatorname{cov}\left(Y^{*}\right)$ is the unique solution of the set of linear equations:

$$
\begin{aligned}
\operatorname{cov}(Y)= & \operatorname{cov}(B)+\sum_{r=1}^{\infty}\left(\mathrm{A}^{r} \hat{\mathcal{B}}(r)+\left[\mathrm{A}^{r} \hat{\mathcal{B}}(r)\right]^{T}\right) \\
& +\mathrm{A} \operatorname{cov}(Y) \mathrm{A}^{T}+Q .
\end{aligned}
$$

The second moment matrix $E\left[Y Y^{T}\right]$ in steady state is the unique solution of the set of linear equations:

$$
\begin{aligned}
E\left[Y Y^{T}\right] & =E\left[B_{0} B_{0}^{T}\right]+\sum_{r=1}^{\infty}\left(\mathrm{A}^{r} \mathcal{B}(r)+\left[\mathrm{A}^{r} \mathcal{B}(r)\right]^{T}\right) \\
& +\mathrm{A} E\left[Y Y^{T}\right] \mathrm{A}+Q_{i j} .
\end{aligned}
$$

Remark 1: Note that the sums both in (8) as well as in (9) are finite since the finiteness for all $i$ of the second moments $b_{i}^{(2)}$ implies that $\mathcal{B}(j)$ are uniformly bounded and since $\|A\|<1$. Note also that if for some $i, b_{i}^{(2)}$ is infinite then it follows directly from (1) that $E\left(\left[Y_{n}\right]_{i}^{2}\right)$ is infinite for all $n>0$ and thus also in the stationary regime.

Proof of Theorem 2. (i) Taking the first moment at stationary regime of (1) we obtain (7).

(ii) To obtain the covariance, we first compute

$$
\begin{aligned}
E[ & \left.\left(A_{0}\left(Y_{0}\right)\right)_{i}\left(A_{0}\left(Y_{0}\right)\right)_{j}\right] \\
= & E\left(E\left[\left(A_{0}\left(Y_{0}\right)\right)_{i}\left(A_{0}\left(Y_{0}\right)\right)_{j} \mid Y_{0}\right]\right) \\
= & E\left(\sum_{k=1}^{N} Y_{0}^{k} \mathrm{~A}_{k i} \sum_{m \neq k} Y_{0}^{m} \mathrm{~A}_{j m}\right) \\
& +E\left(\sum_{k=1}^{N} E\left[\sum_{r=1}^{Y_{0}^{k}} \sum_{s=1}^{Y_{0}^{k}} \xi_{k i}^{(r)} \xi_{k j}^{(s)} \mid Y_{0}^{k}\right]\right) \\
= & \sum_{k=1}^{N} \sum_{m \neq k} \mathrm{~A}_{i k} \mathrm{~A}_{j m} E\left[Y_{0}^{k} Y_{0}^{m}\right] \\
& +E\left(\sum_{k=1}^{N} E\left[\sum_{r=1}^{Y_{0}^{k}} \sum_{s=1, s \neq r}^{Y_{0}^{k}} \xi_{k i}^{(r)} \xi_{k j}^{(s)} \mid Y_{0}^{k}\right]\right)
\end{aligned}
$$

$+E\left(\sum_{k=1}^{N} E\left[\sum_{r=1}^{Y_{0}^{k}} \xi_{k i}^{(r)} \xi_{k j}^{(r)} \mid Y_{0}^{k}\right]\right)$

$$
\begin{aligned}
= & \sum_{k=1}^{N} \sum_{m \neq k} \mathrm{~A}_{i k} \mathrm{~A}_{j m} E\left[Y_{0}^{k} Y_{0}^{m}\right] \\
& +\sum_{k=1}^{N}\left[\left(y_{k}^{(2)}-y_{k}\right)\right] \mathrm{A}_{i k} \mathrm{~A}_{j k}+\sum_{k=1}^{N} y_{k} E\left[\xi_{k i}^{(0)} \xi_{k j}^{(0)}\right] \\
= & \sum_{k=1}^{N} \sum_{m=1}^{N} \mathrm{~A}_{i k} \mathrm{~A}_{j m} E\left[Y_{0}^{k} Y_{0}^{m}\right]+\sum_{k=1}^{N} y_{k} \operatorname{cov}(\xi)_{i j}^{k}
\end{aligned}
$$

and, with $\mathbf{B}_{\mathbf{0}}^{-}:=\left(B_{0}, B_{-1}, B_{-2}, \ldots\right)$ we further compute

$$
\begin{aligned}
E & {\left[\left(Y_{0}\right)_{i} B_{0}^{r}\right]=\sum_{j=0}^{\infty} E\left\{\left[\left(\prod_{i=-j}^{-1} A_{i}^{(-j)}\right)\left(B_{-j-1}\right)\right]_{i} B_{0}^{r}\right\} } \\
& =\sum_{j=0}^{\infty} E\left(E\left\{\left[\left(\prod_{i=-j}^{-1} A_{i}^{(-j)}\right)\left(B_{-j-1}\right)\right]_{i} B_{0}^{r}\right\} \mid \mathbf{B}_{\mathbf{0}}^{-}\right) \\
& =\sum_{j=0}^{\infty} E\left(\left(\mathrm{~A}^{j} B_{-j-1}\right)_{i} B_{0}^{r}\right) \\
& =\sum_{j=0}^{\infty} \sum_{s=1}^{N}\left(\mathrm{~A}^{j}\right)_{i s} \mathcal{B}(j+1)_{s, r}
\end{aligned}
$$

where the last equality follows from (4). Note that the last sum is finite since the finiteness for all $i$ of the second moments $b_{i}^{(2)}$ implies that $\mathcal{B}(j)$ are uniformly bounded and since $\|A\|<1$. Next we compute

$$
\begin{aligned}
E\left[\left(A_{0}\left(Y_{0}\right)\right)_{i} B_{0}^{r}\right] & =E\left[\left(\left(A_{0}\left(Y_{0}\right)\right)_{i} B_{0}^{r} \mid Y_{0}, B_{0}\right]\right. \\
& =\sum_{k=1}^{N} \mathrm{~A}_{i k} E\left[\left(Y_{0}\right)_{k} B_{0}^{r}\right] \\
& =\sum_{j=1}^{\infty}\left(\mathrm{A}^{j} \mathcal{B}(j)\right)_{i, r}
\end{aligned}
$$

We thus obtain

$$
\begin{aligned}
& E\left[Y_{0}^{i} Y_{0}^{j}\right]=E\left[B_{0}^{i} B_{0}^{j}\right]+E\left[\left(A_{0}\left(Y_{0}\right)\right)_{i} B_{0}^{j}\right] \\
& +E\left[\left(A_{0}\left(Y_{0}\right)\right)_{j} B_{0}^{i}\right] \\
& +\sum_{k=1}^{N} \sum_{m=1}^{N} E\left[Y_{0}^{k} Y_{0}^{m}\right] \mathrm{A}_{i k} \mathrm{~A}_{j m}+Q_{i j} \\
& =E\left[B_{0}^{i} B_{0}^{j}\right]+\sum_{r=1}^{\infty}\left(\mathrm{A}^{r} \mathcal{B}(r)+\left[\mathrm{A}^{r} \mathcal{B}(r)\right]^{T}\right)_{i, j} \\
& +\sum_{k=1}^{N} \sum_{m=1}^{N} E\left[Y_{0}^{k} Y_{0}^{m}\right] \mathrm{A}_{i k} \mathrm{~A}_{j m}+Q_{i j}
\end{aligned}
$$

which gives in matrix notation (9). 
We now rewrite (9) as

$$
\begin{aligned}
\operatorname{cov}(Y) & +y y^{T} \\
= & \operatorname{cov}(B)+\hat{B}+\sum_{r=1}^{\infty}\left(\mathrm{A}^{r} \hat{\mathcal{B}}(r)+\left[\mathrm{A}^{r} \hat{\mathcal{B}}(r)\right]^{T}\right) \\
& +\mathrm{A}(I-\mathrm{A})^{-1} \hat{B}+\hat{B}\left(I-\mathrm{A}^{T}\right)^{-1} \mathrm{~A}^{T} \\
& +\mathrm{A} \operatorname{cov}(Y) \mathrm{A}+\mathrm{A} y y^{T} \mathrm{~A}+Q_{i j}
\end{aligned}
$$

We now note that

$$
\begin{aligned}
y y^{T}= & \hat{B}+\mathrm{A}(I-\mathrm{A})^{-1} \hat{B}+\hat{B}\left(I-\mathrm{A}^{T}\right)^{-1} \mathrm{~A}^{T} \\
& +\mathrm{A} y y^{T} \mathrm{~A}
\end{aligned}
$$

which is obtained after some elementary algebra and after substituting $y=(I-\mathrm{A})^{-1} b$. We conclude that $\operatorname{cov}(Y)$ is a solution of (8).

Next, we show uniqueness. Let $Z_{1}$ and $Z_{2}$ be two solutions of (8) and define $Z=Z_{1}-Z_{2}$. Then $Z$ satisfies $Z=\mathrm{A}^{T} Z \mathrm{~A}$. Iterating that we obtain that

$$
Z=\lim _{n \rightarrow \infty} \mathrm{A}^{n} Z\left(\mathrm{~A}^{T}\right)^{n}=0
$$

where the last equality follows since $\|A\|<1$. This implies the uniqueness of the solution for (8). The uniqueness of the solution of (9) is obtained similarly.

\section{B. Example of a correlated processes}

We assume in this Subsection that $B_{n}$ are random vectors whose distribution depends on an underlying ergodic Markov chain $\theta_{n}$ taking values in a finite space $\Theta$. We denote its transition probability by $\mathcal{P}$. Let $\pi$ be the unique steady state probability of the Markov chain. Let $G_{r}^{i}(\theta):=P\left(B_{n}^{i}=r \mid \theta_{n}=\theta\right)$, Let $\hat{G}^{i}$ be a matrix of size $|\Theta| \times \mathrm{Z}^{+}$whose $l r$ th component $\hat{G}_{r}^{i}(l)$ equals $G_{r}^{i}(l) \pi(l)$. Let $J$ be a row vector whose $i$ th entry equals $i, i \in \mathrm{Z}^{+}$ Then for $j>0$, Our goal is to compute the quantities that appear in (8) (in particular $\mathcal{B}(k)$ ).

Lemma 1: In the Markov correlated model described above, we have

$$
[\mathcal{B}(k)]_{i j}=E\left[B_{0}^{i} B_{k}^{j}\right]=J \hat{G}^{i} \mathcal{P}^{k-1}\left[G^{j}\right]^{T} J^{T} .
$$

If we denote by 1 the column vector with appropriate size whose entries are all ones, then we further have:

$$
[\hat{\mathcal{B}}(k)]_{i j}=\left(J-b_{i} \mathbf{1}^{T}\right) \hat{G}^{i} \mathcal{P}^{k-1}\left[G^{j}\right]^{T}\left(J-b_{j} \mathbf{1}\right)^{T} .
$$

where $b_{i}=\sum_{l \in \Theta} E\left[B_{0}^{i} \mid \theta_{0}=l\right] \pi(l) .{ }^{1}$ Moreover.

$$
[\operatorname{cov}(B)]_{i j}=\sum_{\theta \in \Theta} \pi(\theta) \operatorname{cor}[B(\theta)]_{i j}
$$

\footnotetext{
${ }^{1}$ Note that $\left(J-b_{i} \mathbf{1}^{T}\right) \hat{G}^{i}$ is a row vector of dimension $|\Theta|$ whose $l$ th entry equals $E\left[B_{0}^{i}-b_{i} \mid \theta_{0}=l\right] \pi(l)$.
}

where $\operatorname{cor}[B(\theta)]_{i j}=E\left[B_{0}^{i} B_{0}^{j} \mid \theta_{0}=\theta\right]$.

Proof: We have

$$
P\left(B_{0}^{i}=r, B_{k}^{j}=s \mid \theta_{n}=\theta\right)=G_{r}^{i}(\theta) \sum_{\theta^{\prime} \in \Theta}\left[\mathcal{P}^{k-1}\right]_{\theta \theta^{\prime}} G_{s}^{j}\left(\theta^{\prime}\right)
$$

which implies

$$
\begin{aligned}
P & \left(B_{0}^{i}=r, B_{k}^{j}=s\right) \\
& =\sum_{\theta \in \Theta} \pi(\theta) G_{r}^{i}(\theta) \sum_{\theta^{\prime} \in \Theta}\left[\mathcal{P}^{k-1}\right]_{\theta \theta^{\prime}} G_{s}^{j}\left(\theta^{\prime}\right) \\
& =\sum_{\theta \in \Theta} \hat{G}_{r}^{i}(\theta) \sum_{\theta^{\prime} \in \Theta}\left[\mathcal{P}^{k-1}\right]_{\theta \theta^{\prime}} G_{s}^{j}\left(\theta^{\prime}\right) \\
& =\left[\hat{G}^{i} \mathcal{P}^{k-1}\left(G^{j}\right)^{T}\right]_{r s} .
\end{aligned}
$$

Hence $[\mathcal{B}(k)]_{i j}=\sum_{s} \sum_{r} r s\left[\hat{G}^{i} \mathcal{P}^{k-1} G^{T}\right]_{r s}$ which gives (10). The rest is direct.

Next, consider the special case that the $B_{n}^{i}$,s have only values 0 or 1 . Let $p$ and $\hat{p}$ denote the matrices whose $(i, \theta)$ entry equal, respectively, to $p_{\theta}(i):=P\left(B_{n}^{i}=1 \mid \theta_{n}=\right.$ $\theta)$ and $\hat{p}_{\theta}(i):=P\left(B_{n}^{i}=1 \mid \theta_{n}=\theta\right)-P\left(B_{n}^{i}=1\right)$. Let $g$ denote the matrix whose $(i, \theta)$ entry equals $g_{\theta}(i)=$ $\pi(\theta) \hat{p}_{\theta}(i)$. Then (11) simplifies to

$$
\hat{\mathcal{B}}(k)=g \mathcal{P}^{k-1} \hat{p}^{T}
$$

\section{The one dimension case}

We next consider scalar stochastic recursive equations, i.e. $N=1$. $Y_{n}$ in (1) is then a scalar instead of a vector and (2) simplifies to

$$
A_{n}\left(Y_{n}\right)=\sum_{k=1}^{Y_{n}} \xi^{(k)}(n) .
$$

$\xi^{(k)}$ and $\mathrm{A}$ are scalar too with $E\left[\xi^{(k)}(n)\right]=\mathrm{A}$. Theorem 2 simplifies to:

Theorem 3: (i) The first moment of $Y_{n}^{*}$ is given by

$$
E\left[Y_{0}^{*}\right]=\frac{b}{1-\mathrm{A}},
$$

(ii) The variance of $Y_{n}^{*}$ is given by

$$
\begin{aligned}
& \operatorname{var}\left[Y^{*}\right]=E\left[\left(Y^{*}\right)^{2}\right]-\left(E\left[Y^{*}\right]\right)^{2} \\
& =\frac{\operatorname{var}[B]+\sum_{r=1}^{\infty}\left(\mathrm{A}^{r} \hat{\mathcal{B}}(r)+\left[\mathrm{A}^{r} \hat{\mathcal{B}}(r)\right]^{T}\right)+\mathrm{A} b}{1-\mathrm{A}^{2}}
\end{aligned}
$$

Next, we shall further restrict to the Markovian setting of Section III-B. We shall provide an explicit expression for $\sum_{r=1}^{\infty} \mathrm{A}^{r} \mathcal{B}(r)$. 
Lemma 2: In the one dimensional state with the Markov model for correlation, we have

$$
\sum_{r=1}^{\infty} \mathrm{A}^{r} \mathcal{B}(r)=\mathrm{A}\left(J-b \mathbf{1}^{T}\right) \hat{G}[I-\mathrm{A} \mathcal{P}]^{-1} G^{T}(J-b)^{T}
$$

Proof. We get using (11)

$$
\mathrm{A}^{r} \mathcal{B}(r)=\mathrm{A}\left(J-b \mathbf{1}^{T}\right) \hat{G}[\mathrm{~A} \mathcal{P}]^{r-1} G^{T}(J-b)^{T},
$$

$\sum_{r=1}^{\infty}[\mathrm{A} \mathcal{P}]^{r-1}$ is well defined since $|\mathrm{A}|<1$ and since $\mathcal{P}$ is a stochastic matrix. Define $J^{x}$ to be a row vector whose $i$ th entry equals $\min (i, x), i \in \mathrm{Z}^{+}$. Then for any $x>0$, we have by the bounded convergence theorem:

$$
\begin{aligned}
& \sum_{r=1}^{\infty} \mathrm{A}\left(J^{x}-b \mathbf{1}^{T}\right) \hat{G}[\mathrm{~A} \mathcal{P}]^{r-1} G^{T}\left(J^{x}-b\right)^{T} \\
= & \mathrm{A}\left(J^{x}-b \mathbf{1}^{T}\right) \hat{G}[I-\mathrm{A} \mathcal{P}]^{-1} G^{T}\left(J^{x}-b\right)^{T} .
\end{aligned}
$$

(16) is then obtained by the monotone convergence theorem.

We thus obtain the following:

Corollary 1: Consider the scalar case, and consider the Markov model for the correlation process of Section III-B. Then

$$
\begin{aligned}
& \operatorname{var}\left[Y^{*}\right] \\
& =\frac{\operatorname{var}[B]+2 \mathrm{~A}\left(J-b \mathbf{1}^{T}\right) \hat{G}[I-\mathrm{AP}]^{-1} G^{T}(J-b)^{T}+\mathrm{A} b}{1-\mathrm{A}^{2}}
\end{aligned}
$$

Moreover, in the special case that the $B_{n}^{i}$,s have only values 0 or 1 , then we get

$$
\operatorname{var}\left[Y^{*}\right]=\frac{\operatorname{var}[B]+2 \mathrm{~A} g(I-\mathrm{A} \mathcal{P})^{-1} \hat{p}^{T}+\mathrm{A} b}{1-\mathrm{A}^{2}}
$$

\section{THE G/PH/ $\infty$ QUEUE}

We now consider a discrete time $\mathrm{G} / \mathrm{PH} / \infty$ queue. We shall apply in this section the general theory of previous sections in order to compute the steady state moments of some performance measures. We shall then strengthen in the following section the stability results (corresponding to Theorem 1) while relaxing further the statistical assumptions.

\section{A. The model}

Service times: Service times are considered to be i.i.d. and independent of the arrival process. We represent the service time as the discrete time analogous of a phase type distribution: there are $N$ possible service phases. The initial phase $k$ is chosen at random according to some probability $p(k)$. If at the beginning of slot $n$ a customer is in a service phase $i$ then it will move at the end of the slot to a service phase $j$ with probability $P_{i j}$. With probability $1-\sum_{j=1}^{N} P_{i j}$ it ends service and leaves the system at the end of the time slot. Let $\xi^{(k)}(n), k=1,2,3, \ldots$, $n=1,2,3, \ldots$ be i.i.d. random matrices of size $N \times N$. Each of its element can take values of 0 or 1 , and the elements are all independent. The $i j$ th element of $\xi^{(k)}(n)$ has the interpretation of the indicator that equals one if at time $n$, the $k$ th customer among those present at service phase $i$ moved to phase $j$. Obviously, $E\left[\xi_{i j}^{(k)}(n)\right]=P_{i j}$. $P$ is a sub-stochastic matrix (it has nonnegative elements and it's largest eigenvalue is strictly smaller than 1 ), which means that services ends in finite time w.p.1. and that $(I-P)$ is invertible.

Arrivals: Let $B_{n}=\left(B_{n}^{1}, \ldots, B_{n}^{N}\right)^{T}$ be a column vector for each integer $n$, where $B_{n}^{i}$ is the number of arrivals at the $n$th time slot that start their service at phase $i . B_{n}$ is assumed to be a stationary ergodic sequence and that they have finite expectation.

The state and the recursive equation: Let $Y_{n}^{i}$ denote the number of customers in phase $i$ at time $n$. Then $Y_{n}$ satisfies the recursion (1) where $A_{n}$ is given in (2). In particular, $\mathrm{A}=P$ and indeed we have $\|\mathrm{A}\|<1$ so that Assumption A1 hold.

We can thus apply the results of the previous sections to get the first two moments as well as the general distribution at stationary regime.

\section{B. Main results}

Corollary 2: (i) Theorems 1 and 2 hold for the $\mathrm{G} / \mathrm{PH} / \infty$ queue.

(ii) The first and second moments of the number of customers at the system in stationary regime are given respectively by $\mathbf{1}^{T}(I-\mathrm{A})^{-1} b$ and $\mathbf{1}^{T} \operatorname{cov}(Y) \mathbf{1}$, respectively, where 1 is a column vector with all entries 1 's.

Remark 2: We present a simple interpretation of the first moment of the number of customers at the system. Denote by $\lambda$ the expected number of arrivals per slot. Clearly $\lambda=|b|$ where $|b|$ is the sum of entries of the vector $b$. Define $\zeta$ to be the expected service time of an arbitrary customer and let $\rho=\lambda \zeta$. We shall first compute $\zeta$. The $i j$ th element of the matrix $(I-\mathrm{A})^{-1}$ has the interpretation of the total expected number of slots that a customer that had arrived at service phase $j$ spent at state $i$. Thus the $j$ th entry of the vector $\mathbf{1}^{T}(I-\mathrm{A})^{-1}$ has the interpretation of the total expected number of slots that a customer that had arrived at service phase $j$ spent in the system. and let the vector $\beta$ be the vector whose $i$ the entry is $b /|b|$. Then

$$
\zeta=\mathbf{1}^{T}(I-\mathrm{A})^{-1} \beta
$$


$\epsilon>0$ is a parameter that will be varied later in order to

$$
\rho=\left(\mathbf{1}^{T}(I-\mathrm{A})^{-1} \beta\right)|b|=\mathbf{1}^{T}(I-\mathrm{A})^{-1} b,
$$

which is our expression for the first moment of the number of customers at the system. This relation is known to hold in fact for general $G / G / \infty$ queues, see e.g. [6, p. 134].

\section{Departure process}

One can use the same methodology to describe the departure process. To do that, we can augment the system with a new "phase" which we call "d" (for departure), and update the phase transitions as follows:

$$
\begin{aligned}
& \bar{P}_{i j}=P_{i j}, \quad i, j \in\{1, \ldots, N\}, \\
& \bar{P}_{i d}=1-\sum_{j=1}^{N} P_{i j}, i \in\{1, \ldots, N\} \\
& \bar{P}_{d i}=0, \quad i \in\{1, \ldots, N, d\}
\end{aligned}
$$

Quantities corresponding to the new system are denoted by adding a bar. We set $\bar{B}_{n}^{i}=B_{n}^{i}$ for $i=1, \ldots, N$ and $\bar{B}_{n}^{d}=0$ for all integers $n$. Since $P$ is assumed to be substochastic, so is $\bar{P}$. Note that in our new system, only customers in phases $1, \ldots, N$ correspond to those really present in the original system, whereas customers at phase $d$ are already out of the system.

\section{The case of geometric service times}

We now study the special case of geometrically distributed service times. In that case the stochastic recursive equation becomes one dimensional. $Y_{n}$ is a scalar and denotes the number of customers in the system. $\xi_{n}^{(k)}$ has the interpretation of the indicator that the $k$ th customer present at the beginning of time-slot $n$ will still be there at the end of the time-slot. Thus the probability that a customer in the system finishes its service within a time slot is precisely $p=1-\mathrm{A}$. We can now apply directly the results of Theorem 3 .

\section{E. Numerical results}

We consider the following simple scenario. Service times are geometrically distributed, the arrival process depends on a Markov chain as in Subsection III-B, and moreover, there can be either one or no arrival at a time slot.

We consider a Markov chain with two states $\{\gamma, \delta\}$ with transition probabilities given by

$$
\mathcal{P}=\left(\begin{array}{lr}
1-\epsilon p & \epsilon p \\
\epsilon q & 1-\epsilon q
\end{array}\right)
$$

vary the correlations. The steady state probabilities of this Markov chain are

$$
\pi=\left(\frac{q}{p+q}, \frac{p}{p+q}\right) .
$$

Hence

$$
\begin{gathered}
b=E[B]=E\left[B^{2}\right]=\frac{q p_{\gamma}+p p_{\delta}}{p+q} \\
\operatorname{var}[B]=\frac{\left(q p_{\gamma}+p p_{\delta}\right)\left(q\left(1-p_{\gamma}\right)+p\left(1-p_{\delta}\right)\right)}{(p+q)^{2}}
\end{gathered}
$$

Note that $\pi, b, E\left[B^{2}\right]$ and $\operatorname{var}[B]$ do not depend on $\epsilon$.

Applying the first part of Theorem 3 we get the following expression for the expected number of customers in the system in stationary regime:

$$
E\left[Y_{0}^{*}\right]=\frac{1}{1-\mathrm{A}} \times \frac{q p_{\gamma}+p p_{\delta}}{p+q} .
$$

Next, we wish to compute the variance of $Y$. We have

$$
\begin{aligned}
\hat{p}= & {\left[\frac{p\left(p_{\gamma}-p_{\delta}\right)}{p+q}, \frac{q\left(p_{\delta}-p_{\gamma}\right)}{p+q}\right], } \\
g= & \frac{p q\left(p_{\gamma}-p_{\delta}\right)}{(p+q)^{2}}[1,-1], \\
(I-\mathrm{A} \mathcal{P})^{-1}= & \frac{1}{(1-\mathrm{A})(1-\mathrm{A}+\epsilon(p+q) \mathrm{A})} \\
& \times\left(\begin{array}{lr}
1-\mathrm{A}+\epsilon \mathrm{A} q & \epsilon \mathrm{A} p \\
\epsilon \mathrm{A} q & 1-\mathrm{A}+\epsilon \mathrm{A} p
\end{array}\right)
\end{aligned}
$$

We thus obtain

$$
\begin{aligned}
g & (I-\mathrm{A} \mathcal{P})^{-1} \hat{p}^{T} \\
& =\frac{p q\left(p_{\gamma}-p_{\delta}\right)^{2}}{(1-\mathrm{A}+\epsilon(p+q) \mathrm{A})(p+q)^{2}}
\end{aligned}
$$

Remark 3: If we consider $\operatorname{var}\left[Y^{*}\right]$ in (17), we see that the dependence on $\epsilon$ comes only through the term in (20). Moreover, we see that for any value of $\mathrm{A}$, this term, and hence $\operatorname{var}\left[Y^{*}\right]$, decrease with $\epsilon$. Large $\epsilon$ means that the Markov chain alternates rapidly between its two states, which results in a lower overall effect of correlation. (20) can precisely be used to determine this overall effect as it can be viewed of the total weighted sum of correlations $\hat{\mathcal{B}}(k)$, i.e.

$$
g(I-\mathrm{A} \mathcal{P})^{-1} \hat{p}^{T}=\sum_{k=0}^{\infty} g(\mathrm{~A} \mathcal{P})^{k} \hat{p}^{T}=\sum_{k=1}^{\infty} \mathrm{A}^{k-1} \hat{\mathcal{B}}(k)
$$

where we used (13). 
Let $B_{n}=\left(B_{n}^{1}, \ldots, B_{n}^{N}\right)^{T}$ be a column vector for each

As an example, consider the following parameters: $p=$ $q=1, p_{\gamma}=1, p_{\delta}=0.5$. Substituting these parameters in (18), (19) and (20) and plugging these expressions into (17), we obtain the following expressions

$$
\operatorname{var}\left[Y^{*}\right]=\frac{1}{\left(1-\mathrm{A}^{2}\right)}\left(\frac{3}{16}+\frac{2 \mathrm{~A}}{1-\mathrm{A}+2 \epsilon \mathrm{A}}+\frac{3}{4} \mathrm{~A}\right) .
$$

In Fig. 1 we plot the variance of the steady state number of customers, $\operatorname{var}\left[Y^{*}\right]$, while varying $\epsilon$ and A.

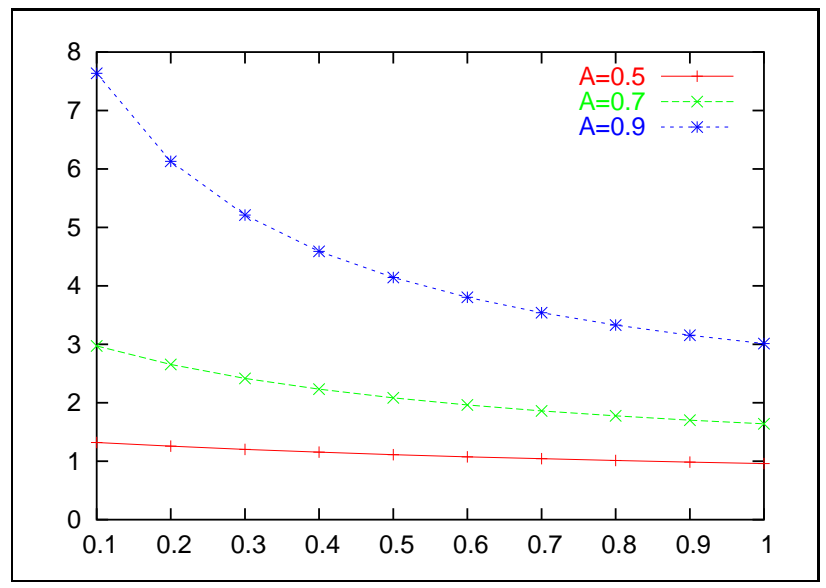

Fig. 1. $\operatorname{var}[Y *]$ as a function of $\epsilon$ and of $\mathrm{A}$

Recall that for a fixed A, the expectation of $Y^{*}$ does not depend on $\epsilon$. The variance of $Y^{*}$ on the other hand is seen to be quite sensitive to the correlation between the $B_{n}$ 's as determined by the parameter $\epsilon$. This sensitivity is seen to increase as $A$ increases and sensitivity is largest when $A$ approaches 1 . As already mentioned in Remark 3 we see that $\epsilon=1$ gives the smallest value of $E\left[Y^{*}\right]$ and that $E\left[Y^{*}\right]$ increases as $\epsilon$ decrease. For $\mathrm{A}=0.5$ we get a difference of around $30 \%$ between the lowest and the largest value of $\epsilon$, where as form $\mathrm{A}=0.9$ we obtain a difference of $250 \%$.

\section{F. Extension to a network}

Consider now $M$ stations, each with infinite number of servers. The service time at station $i$ has a set $\mathcal{N}_{i}$ of $N_{i}$ phases. Let $N=N_{1}+\ldots+N_{M}$. For any $j=1, \ldots, N$ let $s(j)$ denote the station to which $j$ corresponds, i.e. if $j \in \mathcal{N}_{i}$ then $s(j)=i$.

If at time $n$ a customer was at phase $j$ in station $s(j)$ then it either moves to another phase at the same station or moves to another phase in another station; the next phase $k$ (either at the same station or at another one) is chosen with probability $P_{j k}$; with probability $1-\sum_{k=1}^{N} P_{j k}$ the customer leaves the system. Again we assume that the choice of next phase are independent. integer $n$, where $B_{n}^{i}$ is the number of arrivals at the $n$th time slot that start their service at phase $i$ in station $s(i)$. $B_{n}$ is assumed to be a stationary ergodic sequence.

With this description we see that we can identify the whole network as a single server station problem with infinite number of servers and with $N$ phases. Thus we can apply all previous results.

\section{Residual ACtivity time IN the $G / G / \infty$ QUeUe}

Define the residual activity time at a given instant as the total time till the system empties from that instant onwards if new arrivals do not occur.

We shall analyze in this section the residual activity of a $\mathrm{G} / \mathrm{G} / \infty$ queue under weaker statistical assumptions than those used so far. We shall obtain the existence of a stationary regime as well as convergence to it in the coupling-convergence sense (see e.g. Borovkov [7], [8]).

a) The model: The $n$th arrival event occurs at time $T_{n}$ : a batch of $B_{n}$ customers arrive. Denote $\tau_{n}=T_{n+1}-$ $T_{n}$; they replace the fixed slots we had before. Let $\sigma_{n}$ be the largest service time required among the $B_{n}$ customers that arrive at time $T_{n}$. We shall assume that the joint sequence $\left(\tau_{n}, \sigma_{n}\right)$ is stationary ergodic and that $E\left[\tau_{0}\right]$ and $E\left[\sigma_{0}\right]$ are finite and strictly positive. $\sigma_{n}$ in particular, need not have a "phase type distribution" as before. Let $V_{n}$ be the residual activity time just before $T_{n}$. Then $V_{n}$ can be written recursively as:

$$
V_{n+1}=\left(\max \left(V_{n}, \sigma_{n}\right)-\tau_{n}\right)^{+}
$$

where $(x)^{+}:=\max (x, 0)$.

Iterating this relation gives:

$$
\begin{aligned}
& V_{n+2} \\
& \quad=\left(\max \left\{\left[\max \left(V_{n}, \sigma_{n}\right)-\tau_{n}\right]^{+}, \sigma_{n+1}\right\}-\tau_{n+1}\right)^{+} \\
& =\max \left(\max \left(V_{n}, \sigma_{n}\right)-\tau_{n}-\tau_{n+1}, \sigma_{n+1}-\tau_{n+1}, 0\right) .
\end{aligned}
$$

Further iterating directly yields:

$$
V_{n+k}=\max \left(Z_{n}, Z_{n+1}, \ldots ., Z_{n+k-1}, 0\right)
$$

where

$$
\begin{gathered}
Z_{n}=\max \left(V_{n}, \sigma_{n}\right)-\sum_{i=0}^{k-1} \tau_{n+i} \\
Z_{n+j}=\sigma_{n+j}-\sum_{i=j}^{k-1} \tau_{n+i}, \quad j=1, \ldots, k-1 .
\end{gathered}
$$


b) Stationary solution: We use the Loynes' type scheme [16] to obtain the stationary regime and the convergence to it.

Theorem 4: $V_{n}$ converges a.s. to a unique stationary regime that is given by

$$
V_{n}^{*}:=\left(\max _{j<n}\left[\sigma_{j}-\sum_{i=j}^{n-1} \tau_{i}\right]\right)^{+} .
$$

from any initial $V_{0}$. Moreover $V_{n}^{*}$ is $P-a . s$. finite.

Proof: Define on the same probability space as the process $V_{n}$ the shifted processes $V_{n}^{[m]}$, where $m$ are integers:

$V_{-m}^{[m]}=0, \quad V_{n+1}^{[m]}=\left(\max \left(V_{n}^{[m]}, \sigma_{n}\right)-\tau_{n}\right)^{+}, n \geq-m$.

Then as before, we can write for $n>-m$ :

$$
V_{n}^{[m]}=\left(\max _{-m \leq j<n}\left[\sigma_{j}-\sum_{i=j}^{n-1} \tau_{i}\right]\right)^{+}
$$

which monotonely increases to the sequence $V_{n}^{*}$ given in (21). Clearly $V_{n}^{*}$ is a stationary ergodic process. We shall show that it is $P-a . s$. finite. Indeed, since $\left(\tau_{n}, \sigma_{n}\right)$ is stationary ergodic, the Cesaro sums converge to the expectation $P-a . s$. and hence there is some R.V. $J_{0}$ which is finite $P-a . s$. such that for all $j>J_{0}$,

$$
\sigma_{-j}<j E\left[\tau_{0}\right] / 3 \text { and } \sum_{i=-j}^{-1} \tau_{i}>j 2 E\left[\tau_{0}\right] / 3
$$

Hence the term in brackets in (21) is negative for all $-j>$ $J_{0}$ so that $V_{0}^{*}$ is finite $P-a . s$. Due to stationarity this is true for $V_{n}^{*}$ for all $n$.

c) Coupling: We show that for any initial value $V_{0}$ there is a time $N_{0}$ which is finite $P-a . s$. such $V_{n}$ coincides with $V_{n}^{*}$ for all $n>N_{0}$. Indeed, fix $V_{0}$ and define

$$
N_{0}:=\inf \left\{l: \max \left(V_{0}, V_{0}^{*}\right)<\sum_{i=0}^{l-1} \tau_{i}\right\} .
$$

$N_{0}$ is clearly finite $P-$ a.s. due to the ergodicity of $\tau_{i}$. Moreover, it is clear from the explicit expressions we have for $V_{0}$ and for $V_{0}^{*}$ that they coincide for $n>N_{0}$. Uniqueness of the stationary regime follows from the fact that coupling has been established for arbitrary initial state.

Remark 4: Our construction establishes in fact that we have strong coupling convergence in the sense of [7], [8].

Remark 5: A stability result is already given in [6, p. 133] for a general G/G/ $\infty$ queue. Namely, it is shown that $V_{0}$ is finite almost surely but the form of the stationary regime and the convergence results are not given.

\section{CONCLUDING COMMENTS}

In this paper we have studied and used stochastic recursive equations to investigate the discrete infinite server queue with batch arrivals where the size of the batches follow a general stationary ergodic process. We obtained explicit expressions for the first and second moments of the state variables appearing in the stochastic recursive equations and applied them to solve the infinite server queue problem. We proposed then more specific Markov models for correlation that further simplify the expressions for the first two moments. We extended the results of the infinite server queue to a whole network of such queues.

Other stochastic recursive equations have been used to study the stability of the queue under even more general probabilistic assumptions and convergence to a unique stationary regime has been established.

The simple explicit expressions obtained makes our results appealing to various applications of the infinite server queue. For example, they can be used to represent the first and second moments of the number of connected mobiles at an arbitrary location in the one dimensional adhoc network of [17], using the equivalence between the ad-hoc network and an infinite server queue given in [17].

\section{ACKNOWLEDGEMENT}

This work was supported by the EURO NGI network of excellence.

\section{REFERENCES}

[1] S. R. Adke and V. G. Gadag, "A new class of branching processes", Branching Processes: Proceedings of the First World Congress, C.C.Heyde (Editor), 1-13, Springer Lecture Notes 99, 1995.

[2] E. Altman, "Stochastic recursive equations with applications to queues with dependent vacations", Annals of Operations Research, 112(1): 43-61; Apr, 2002.

[3] E. Altman and A. Hordijk, "Applications of Borovkov's Renovation Theory to Non-Stationary Stochastic Recursive Sequences and their Control", Advances of Applied Probability 29, pp. 388413, 1997.

[4] E. Altman, T. Jimenez, D. Kofman, "DPS queues with stationary ergodic service times and the performance of TCP in overload", Proceedings of IEEE Infocom, Hong-Kong, March 2004.

[5] E. Altman, P. Konstantopoulos, Z. Liu, "Stability, Monotonicity and Invariant Quantities in General Polling Systems", Queueing Systems 11 , pp. 35-57, 1992

[6] F. Baccelli and P. Brémaud, Elements of Queueing Theory, Springer, second edition, 2003.

[7] A. A. Borovkov, Asymptotic Methods in Queueing Theory, John Wiley \& Sons, 1984 (translated from Russian).

[8] A. A. Borovkov and S. G. Foss, "Stochastically recursive sequences and their generalizations", Siberian Advances in Mathematics, 2, No. 1, pp. 16-81, 1992.

[9] A. Brandt, "The stochastic equation $Y_{n+1}=A_{n} Y_{n}+B_{n}$ with stationary coeffi cients", Advances in Applied Probability, Vol. $18,1986$. 
[10] A. Brandt, P. Franken and B. Lisek, Stationary Stochastic Models, Akademie-Verlag, Berlin, 1992.

[11] P. Glasserman and D. D. Yao, "Stochastic vector difference equations with stationary coeffi cients", J. Appl. Prob., Vol 32, pp 851866, 1995.

[12] R. Groenevelt and E. Altman, "An alternating priority server with correlated and cross correlated vacations", manuscript.

[13] A. Jean-Marie and P. Robert, On the transient behavior of the processor sharing queue, QUESTA 17, No. 1-2, pp. 129-136, 1994.

[14] H. Kameda and Y. Zhang, "Uniqueness of the solution for optimal static routing in open BCMP queueing networks", Math. Comput. Modeling 22, No. 10-12, 119-130, 1995.

[15] M. Kruntz and A. Makowski, 'Modeling video traffi c using M/G/infi nity input processes: A compromise between Markovian and LRD models," IEEE Journal on Selected Areas in Communications (JSAC), pp. 733-748, Vol. 16, No. 5, June 1998.

[16] R. Loynes, "The stability of a queue with non-independent interarrival and service times", Proc. Cambr. Phil. Soc. 58, No. 3, pp. 497-520, 1962.

[17] D. Miorandi and E. Altman, 'Connectivity in Ad-Hoc Networks: a Queueing Theoretical Approach", Proceedings of WiOpt workshop, Cambridge, UK, March 2004. 